\title{
Avoiding diagnostic errors in psychosomatic medicine: a case series study
}

\author{
Atsuko Koyama ${ }^{1 *}$ D, Yoichi Ohtake ${ }^{2}$, Kanae Yasuda ${ }^{3}$, Kiyohiro Sakai ${ }^{1}$, Ryo Sakamoto ${ }^{1}$, Hiromichi Matsuoka', \\ Hirokuni Okumi ${ }^{1}$ and Toshiko Yasuda ${ }^{1}$
}

\begin{abstract}
Background: Non-organic lesions or diseases of unknown origin are sometimes misdiagnosed as "psychogenic" disorders or "psychosomatic" diseases. For the quality of life and safety of patients, recent attention has focused on diagnostic error. The aim of this study was to clarify the factors that affected misdiagnoses in psychosomatic medicine by examining typical cases and to explore strategies that reduce diagnostic errors.

Case presentation: The study period was from January 2001 to August 2017. The data of patients who had visited the Department of Psychosomatic Medicine, Kindai University Hospital and its branches, Sakai Hospital and Nihonbashi Clinic, were collected. All patients were aged 16 years or over. Multiple factors, such as age, sex, presenting symptoms, initial diagnosis, final diagnosis, sources of re-diagnosis and types of diagnostic errors were retrospectively analyzed from the medical charts of 20 patients. Among them, four typical cases can be described as follows. Case 1; a 79-year-old woman, initially diagnosed with psychogenic vomiting due to depression that was changed to gastric torsion as the final diagnosis. Case 2; a 24-year-old man, diagnosed with an eating disorder that was later changed to esophageal achalasia. Case 10; a 60-year-old woman's diagnosis changed from conversion disorder to localized muscle atrophy. Case 19; a 68-year-old man, appetite loss from depression due to cancer changed to secondary adrenal insufficiency, isolated ACTH deficiency (IAD).

Conclusion: This study showed that multiple factors related to misdiagnoses were combined and had a mutual influence. However, they can be summarized into two important clinical observations, diagnostic system-related problems and provider issues. Provider issues contain mainly cognitive biases such as Anchoring, Availability, Confirmation bias, Delayed diagnosis, and Representativeness. In order to avoid diagnostic errors, both a diagnostic system approach and the reduction of cognitive biases are needed. Psychosomatic medicine doctors should pay more attention to physical symptoms and systemic examination and can play an important role in accepting a perception of patients based on a good, non prejudicial patient/physician relationship.
\end{abstract}

Keywords: Diagnostic error, Psychosomatic medicine, Psychosomatic disease, Cognitive bias

\section{Background}

Psychosomatic medicine was established in Japan in 1996 as one of the specific medical fields in which "psychosomatic disorders" are dealt with, and it has been developing widely in both Japan and Germany. The department of psychosomatic medicine is classified as part of internal

\footnotetext{
* Correspondence: a-koyama@med.kindai.ac.jp

${ }^{1}$ Department of Psychosomatic Medicine, Faculty of Medicine, Kindai

University, 377-2, Ohno-higashi, Osaskasayama City, Osaka 589-8511, Japan

Full list of author information is available at the end of the article
}

medicine, not psychiatry, and attends to patients complaining of physical symptoms due to psychosocial distress.

Psychosomatic disorders are defined as physical diseases whose onset and course are closely related to psychosocial factors and contain both organic and functional disorders [1]. The diagnosis of true "psychosomatic diseases" should be based on strict differential diagnoses, including functional disorders. However, non-organic lesions or diseases of unknown origin are sometimes diagnosed as 
"psychogenic" disorders or "psychosomatic" diseases. These problems are becoming more prominent according to an increase in the number of patients with psychosomatic diseases. The reduction of diagnostic error is an important goal for the quality of life and safety of patients. The aim of this study was to clarify the factors that affected misdiagnoses in psychosomatic medicine by examining typical cases and to explore strategies that reduce diagnostic errors.

\section{Material and methods}

\section{Patients}

The inclusion criteria for this study were as follows:

1. Patients who had visited the Department of Psychosomatic Medicine, Kindai University Hospital and its branches, Sakai Hospital and Nihonbashi Clinic, from January 2001 to August 2017.

2. Patients who were considered to have physical symptoms due to psychosocial distress at their first visit.

3. Aged 16 years or over, because our department is associated with internal medicine and only provides care for those of high school age or older.

The exclusion criteria were as follows:

1. Patients with primary psychiatric diseases, for example having hallucination and/or delusion.

2. Patients who were unwilling to participate and contacted us to refuse participation.

\section{Design and setting}

This was planned as a case series study.

All patients who visited our department for the first time filled out a systemic medical questionnaire including their demographic background, subjective physical complaints, and psychological distress, after which semistructured interviews were performed by doctors.

During the study period, all items assessed during routine clinical practice were extracted from the patients' medical charts. Multiple factors, such as age, sex, presenting symptoms, initial diagnosis, final diagnosis, sources of re-diagnosis, types of diagnostic errors, and the interval between an initial diagnosis and a final diagnosis were retrospectively analyzed from the medical charts of $20 \mathrm{pa}$ tients. The interval was classified into six categories; less than 1 month, 1 to 3 months, 3 to 6 months, 6 to 12 months, 12 to 24 months, and more than 24 months.

The co-authors gathered cases in which the final diagnosis was significantly different than the initial diagnosis. Almost all cases, except for cases 3 and 20, were referred from another hospital or another department in Kindai University Hospital or Sakai Hospital that reported their initial diagnosis. As for cases 3 and 20, both the initial and final diagnosis were made in our department. Each main doctor in the cases made a true final diagnosis through systematic examination, but in some cases a heuristic method helped determine the diseases. Two doctors independently examined the process of diagnosis and judged the type of diagnostic error. After discussing their findings, their consensus on the type of diagnostic error was adopted.

\section{Results}

\section{Demographic and clinical characteristics}

Approximately 2200 new patients come to Kindai University Hospital and its branches, Sakai Hospital and Nihonbashi Clinic, every month, with 20 to 25 of them visiting the Department of Psychosomatic Medicine. Twenty cases were eligible for this study according to the inclusion criteria.

Detailed patient characteristics are listed in Table 1, which contains the case number, age, sex, symptoms, initial diagnosis, final diagnosis, sources of re-diagnosis, types of diagnostic errors, and the interval between the initial diagnosis and the final diagnosis. The age ranged from 24 to 81 years and the sex ratio was 14 male to 6 female. In comparison, the sex ratio of the patients in our department is 1 male to $2.5 \sim 3$ female.

As an initial diagnosis, three cases were diagnosed with "psychogenic" diseases and six with "psychosomatic" diseases. In addition, two cases were diagnosed with conversion disorder and nine with depressive state. As for the final diagnosis, seven cases were identified by blood examination and detailed hormonal test as having endocrinologic diseases. Another seven cases were diagnosed with neurological diseases after follow-up of the clinical course and brain MRI. Adequate examinations were effective in making the true diagnoses. For example, radiography instead of endoscopy in cases 1 and 2, targeted examination such as the acid-fast bacteria stain in case 4 , and an antibody test in case 6 .

The interval between the initial diagnosis and the final diagnosis ranged from less than 1 month to more than 24 months. The most difficult case in this study was case 10 .

\section{Case presentation}

There were several types of diagnostic errors and differences in the clinical courses. Among the twenty cases, four typical, characteristic cases that are educational and that contain the five representative types of diagnostic errors identified in this study will be described in detail. These cases show give a number of lessons as to the path taken from an initial misdiagnosis to a final, true diagnosis. 

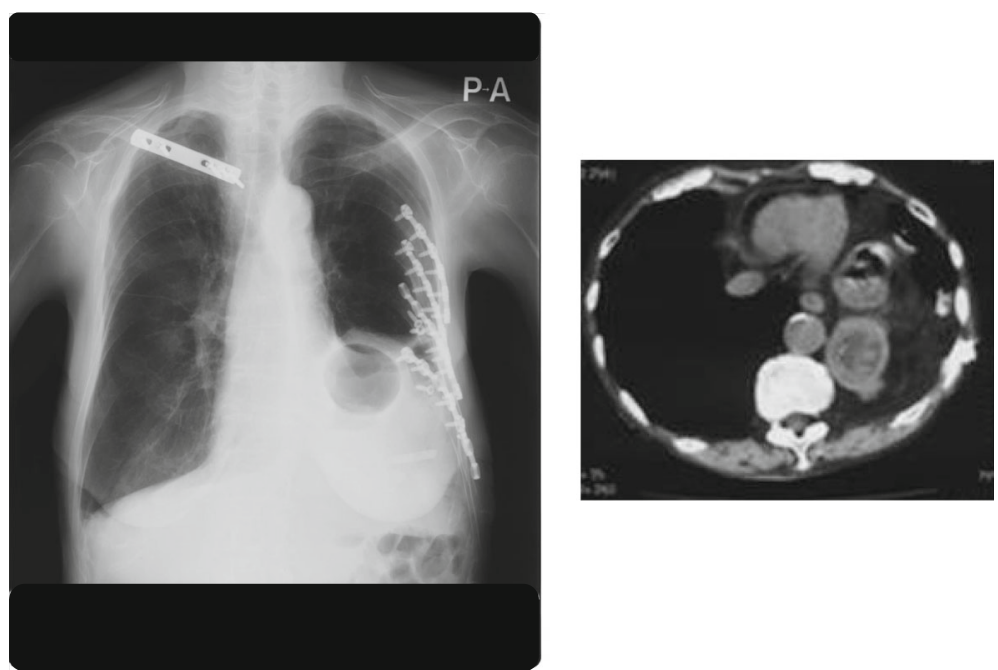

Fig. 1 Chest X-ray and chest CT of case 1 Chest X-ray (left) and chest CT (right) show a left diaphragm hernia. The left diaphragm deviates upwards into the thoracic cavity and gastric gas is seen in the gastric body

food successfully, so it was not self-induced vomiting. The esophageal radiography showed esophageal achalasia (Fig. 3). Transendoscopic myotomy mitigated his symptoms.

Final diagnosis: esophageal achalasia.

\section{Case 10}

\section{Initial diagnosis: Conversion disorder}

A 60-year-old woman presented with difficulty in raising her right arm. For the previous year she had felt weakness and numbness in her right arm. During that time, she moved to her son's home and lost contact with her old friends. Her relationship with her son's wife was worsening and she avoided meals that her son's wife cooked. A specific examination at the department of neurology revealed no abnormality. She was referred to our department with a diagnosis of conversion disorder.

The symptom was gradually progressive and sometimes improved, but at one point improved and worsened on the same day. Several months before the final diagnosis the symptom progressed markedly. When she complained of slight back pain and weakness, we examined her whole body again and found localized muscle atrophy in the right area of her back. We consulted the neurologist again and she was diagnosed with localized muscle atrophy after an electromyogram and muscle biopsy. There is no radical treatment for this problem, so
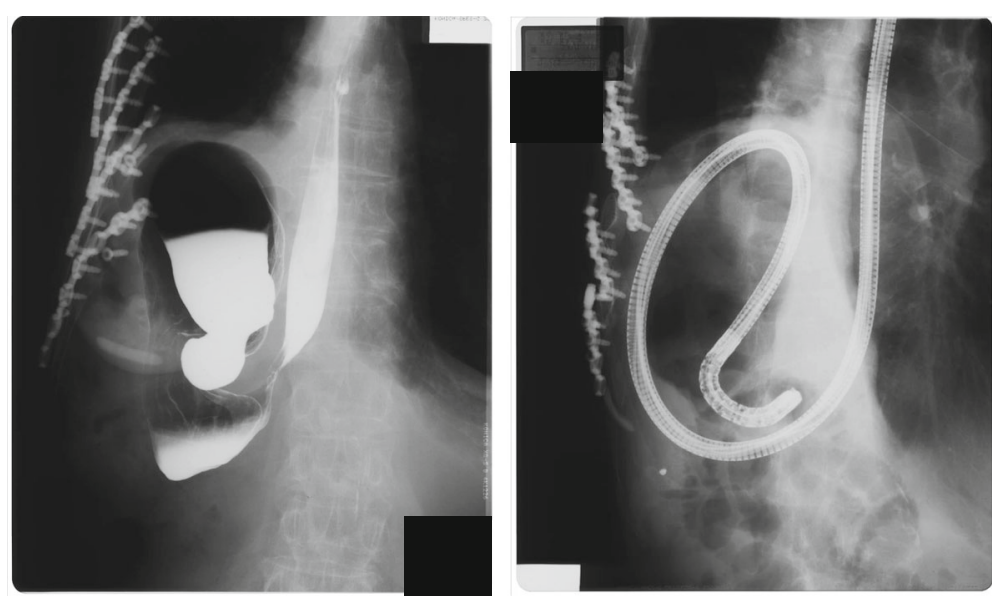

Fig. 2 The upper gastrointestinal radiography and the upper gastrointestinal endoscope examination of case 1 The upper gastrointestinal radiography (left) shows gastric torsion with an adhesion of the gastric body in the upper side rather than the gastric fornix. The upper gastrointestinal endoscope examination (right) shows the torsional axis and position of the gastric body 


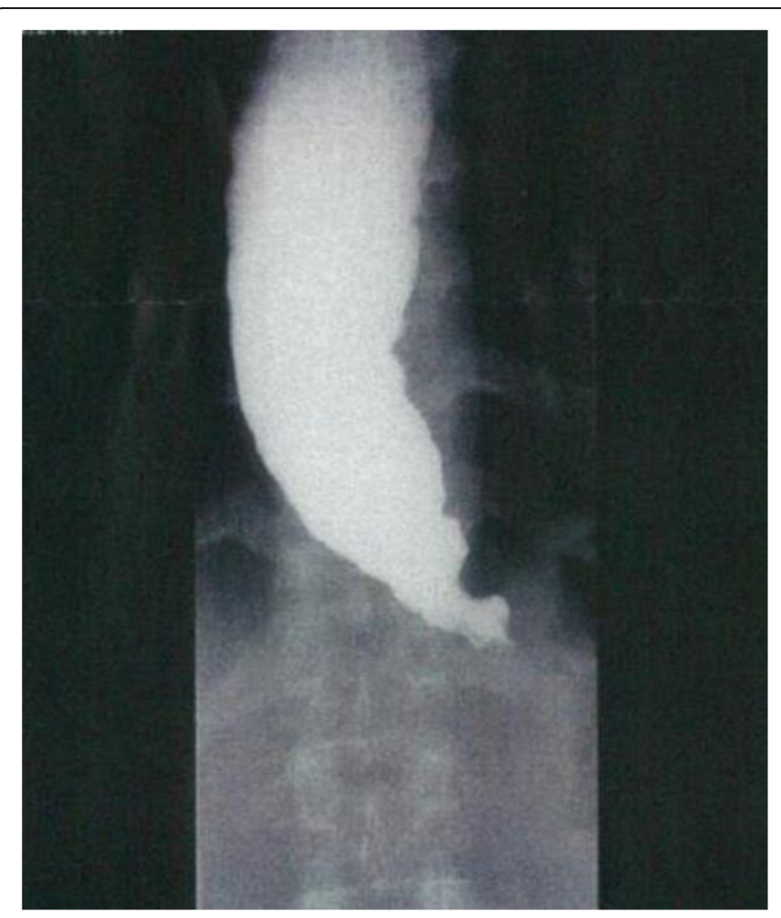

Fig. 3 The esophageal radiography of case 2. The esophageal radiography shows dilation of the esophagus and a "bird-beak" appearance, which are characteristics of esophageal achalasia

she received physical therapy and was kept under observation.

Final diagnosis: localized muscle atrophy.

\section{Case 19}

\section{Initial diagnosis: Depression due to cancer (appetite loss)}

A 68-year-old man presented with symptoms of appetite and weight loss. He suffered from gastric cancer and had had a partial gastrectomy three years previously. He recovered from it and his activity of daily living was normal. However, over the three months before reporting to the hospital, after suffering a common cold he gradually lost his appetite and weight (from 58 to $48 \mathrm{~kg}$ ). He could consume only semisolid food, such as tofu, and a small amount of water, and stayed in bed almost all day long. Upper gastrointestinal endoscope examination, abdominal CT, and tumor marker were normal at the department of gastroenterology. Medicine for gastrointestinal movement did not improve his symptoms. He was referred to our department with a diagnosis of depression due to cancer.

His general condition gradually worsened and he was finally admitted due to dehydration. On the 1 st hospital day, serum $\mathrm{Na}$ was $135 \mathrm{mmol} / \mathrm{L}$ due to dehydration. After drip infusion and tubal feeding for several days, it was found that serum $\mathrm{Na}$ was very low $(119 \mathrm{mmol} / \mathrm{L})$. In the next step, endocrinological laboratory studies showed very low serum and plasma levels of adrenocorticotropic hormone $(\mathrm{ACTH})(<2.1 \mathrm{pg} / \mathrm{ml})$ and cortisol $(0.4 \mu \mathrm{g} / \mathrm{dl})$. Following dynamic tests for pituitary hormone secretion in response to combined stimulation with corticotropin-releasing hormone $(\mathrm{CRH})$, thyrotropinreleasing hormone $(\mathrm{TRH})$, luteinizing hormone-releasing hormone (LH-RH), and growth hormone-releasing hormone $(\mathrm{GH}-\mathrm{RH})$ revealed a blunted response of ACTH. Thus, the patient was diagnosed with isolated ACTH deficiency (IAD). The replacement of hydrocortison 15-20 mg/day improved his condition, he gradually became able to have meals, and he was finally discharged.

Final diagnosis: secondary adrenal insufficiency, isolated ACTH deficiency (IAD).

\section{Discussion}

To summarize the characteristics of the 20 patients, their ages ranged from 24 to 81 years, which seems rather high considering that our department is associated with internal medicine and our hospital only treats patients aged 16 years and over. No age-related factors were found in this study; however, older patients might have a tendency to suffer from complication of multiple diseases due to aging, which makes it difficult to reach a true simple diagnosis.

As for sex-specific features, the reason why there were more male than female patients in this study is unknown. However, the difference in prevalence by sex might give an important hint for reconsidering the diagnostic process. For example, despite the incidence of eating disorder being much higher in female patients, the patient in case 2 was a young male, which led to a more prudent clinical assessment. Therefore, it is important to consider the prevalence of diseases as they relate to age and sex in order to make a correct diagnosis.

This study shows that multiple factors related to misdiagnosis were combined and had a mutual influence. However, they can be summarized into two important clinical observations, diagnostic system-related problems and provider issues [2-5].

\section{Cause of diagnostic errors Diagnostic system-related problems}

Previous studies showed that clinical reasoning is based on two systems. System 1 is an intuitive process based on heuristics [6, 7], and System 2 is an analytical process. Each system has both advantages and disadvantages, as summarized in Table 2 . Both systems can interact and/or override each other [8]. Although System 1 seems to be prone to failure, it has been verified that the two processes are equally effective [9].

In this study, both systems were used, however, the doctors ranged from a novice who preferred System 2 to an expert who is good at System 1, and the interaction of both systems might not have functioned effectively. 
Table 2 Comparison of System 1 and System 2: Types of clinical reasoning

\begin{tabular}{lll}
\hline & Intuitive process & Analytical process \\
& System 1 & System 2 \\
\hline Examples & Heuristics & Algorithm \\
& Pattern recognition & Hypothetical-deductive \\
Feature & Snapshot diagnosis & Comprehensive diagnosis \\
& Unconscientious & Conscientious \\
Advantages & Faster & Scientific \\
& Efficient & Analytical \\
Disadvantages & Biases & Slower \\
\hline
\end{tabular}

\section{Provider issues}

Provider issues contain mainly cognitive biases [3-5], failures in perception and failed heuristics due to lack of medical knowledge and/or clinical experience [5, 10]. Previous studies described several types of cognitive biases, such as Anchoring, Availability, Confirmation bias, Delayed diagnosis, and Representativeness [5, 8, 11, 12], as in Table 1.

Anchoring is the tendency to be affected by an initial impression that is not adjusted by later information. This was seen in cases 1,2 , and 7 .

Availability is the tendency to judge diagnosis by recent experience and the memory of diseases. This was seen in cases 2, 5, 6, 7, 17, 20.

Confirmation bias is the tendency to seek data/evidence to support a diagnosis and to cast away data/evidence that refutes it. This was seen in cases 3, 8, 9, 16, and19.

Delayed diagnosis means that long-term observation is necessary until a true diagnosis is made. This was seen in cases 3, 4, 9, 10, 12, and 20 .

Representativeness is the tendency to look for prototypical manifestations of diseases and to miss atypical variants. This was seen in cases 8,11 , and 13-19.

\section{Strategies to reduce diagnostic errors}

Based on this study, several measures were found that would reduce diagnostic errors.

\section{Diagnostic system improvement}

First, There are several proposals for system level improvement, for example using checklists $[13,14]$ and '12 tips' [15]. Moreover, interventions that use decision-making skills, such as computer searching engines, diagnostic tools on the internet, and facilitating access to information, second opinions, and specialists might be useful [10].

\section{Provider issues}

Next, several issues were found in this study at the individual level.
1) Doctors can never make a true diagnosis of diseases that do not come to mind, for example the endocrine disorders in cases 14-19. Even if incidence and prevalence are very low [16], clinicians should take into account the possibility of rare diseases and their characteristics, such as the IAD in cases 16 and 19. Targeted hormonal examinations are essential for diagnosing ACTH deficiency, severe growth hormone hypoplasia, and diabetes insipidus by lymphocytic hypophysitis. For this issue, the improvement of medical knowledge and diagnostic skill through systematic medical education is essential [8].

2) It is important that trivial abnormal findings should not be ignored. Detailed analysis of examination data is also necessary, as seen in cases $5-9,11,13,14,16$, 18 , and 19.

3) Long term observation is necessary to make a true diagnosis [14], for example, neuromuscular diseases, such as the localized muscular atrophy and amyotrophic lateral sclerosis of cases $3,4,9,10,12$, and 20. This is related to Delayed diagnosis. The most difficult case in this study was case 10 , and the time it took to reach a final, correct diagnosis was more than 24 months. The initial specific examination in the Department of Neurology revealed no abnormality and muscle atrophy was not found, therefore it took a long time until the symptoms progressed markedly and muscle atrophy became obvious.

4) Easy judgement of "psychogenic" diseases and "depressive state" should be avoided, as in cases 1, 5, 6,7 , and10-19. For example, IAD can mimic depressive disorder due to the symptoms of appetite loss and hypoactivity, as in cases 16 and 19 [17]. Several previous studies suggest that misdiagnosis of depression in primary care outpatients occurs fairly often, and even in educational general hospitals non-psychiatric house staff frequently misdiagnose psychiatric disorders [18]. This can be improved by psychiatric training/education $[19,20]$ and using the screening instruments mentioned previously in I. Diagnostic system improvement.

5) If an organic disorder is excluded, there is a possibility of a functional disorder. In cases 1 and 2 the mucosal surface was intact during endoscopic examination, however, there was a functional disorder, such as esophageal achalasia. The definition of psychosomatic diseases contains both organic and functional physical disorders, such as functional somatic syndrome, functional dyspepsia and irritable bowel syndrome. It is the principle of the diagnosis of psychosomatic diseases.

6) Physician overconfidence [21] and emotional reactions to patients [22] lead to misdiagnosis. 
Information from former doctors should be verified, without prejudice, as in cases 1 and 2. The correction of cognition bias by Availability is needed. It is important to include the patient perspective [23] and for patients to be "co-producers" in making a safer diagnostic process [23]. Previous studies showed that a semi-structured interview and the use of positive criteria are effective [24]. Building a good relationship between doctors and patients and cultivating the therapeutic self and self-esteem are also fundamental in psychosomatic medicine.

\section{Limitations}

The present study has several limitations.

First, this study was based on consultation cases only in our hospital. Further studies are needed to clarify factors related to the misdiagnosis of patients visiting departments of psychosomatic medicine throughout Japan.

Second, there is difficulty in discerning the exact factors of misdiagnosis. Clinical reasoning is based on multiple aspects and it is impossible that all clinicians in this study could recall the exact situations and the cause of diagnosis due to time overlay and differences in their clinical knowledge.

Third, there is the inevitable bias of any retrospective analysis in which the outcome is known.

Although our study has several limitations, some highly suggestive results can be regarded as helpful information for clinical psychosomatic practice and for identifying topics for future studies. In order to elucidate the causes of diagnostic errors and to improve strategies to promote psychosomatic medicine, further research addressing the present study's limitations is necessary.

\section{Conclusion}

There is a high possibility of misdiagnosis among patients diagnosed with "psychogenic" disorders or "psychosomatic" diseases. In order to avoid diagnostic errors, both a diagnostic system approach and the reduction of cognitive biases are needed. Psychosomatic medicine doctors should pay additional attention to physical symptoms and systemic examination and can play an important role by adopting a perception of patients based on a good, non-prejudicial patient/physician relationship.

\section{Abbreviations}

ACTH: Adrenocorticotropic hormone; $\mathrm{CRH}$ : Corticotropin-releasing hormone; $\mathrm{GH}-\mathrm{RH}$ : Growth hormone-releasing hormone; IAD: Isolated ACTH deficiency; $\mathrm{LH}-\mathrm{RH}$ : Luteinizing hormone-releasing hormone; TRH: Thyrotropin-releasing hormone

\section{Acknowledgements}

We are grateful to the patients who participated in the study and to Michael Likoycheong for proofreading this article.

\section{Funding}

This research did not receive any specific grants from funding agencies in the public, commercial, or not-for-profit sectors.

Availability of data and materials

Data sharing is not applicable to this article as no datasets were generated or analyzed during the current study.

\section{Authors' contributions}

AK conceived the study and participated in its design. YO participated in its design and was a major contributor to writing the manuscript. AK and YO examined the process of diagnosis and judged the types of diagnostic errors. After discussion, their consensus on the type of diagnostic error was adopted. All authors were involved in the management of these patients and the preparation of the case reports. All authors read and approved the final manuscript.

Ethics approval and consent to participate

This study was approved by the ethics committee of Kindai University, Faculty of Medicine (No. 27-004). We were careful not to infringe on patient privacy and publicly displayed information regarding this study on the homepage of our department (http://www.kindai-psychosomatics.com/) so that those unwilling to participate could contact us to refuse participation.

\section{Consent for publication}

We also publicly displayed information regarding this study on the homepage of our department (http://www.kindai-psychosomatics.com/) so that those unwilling to have their personal data published could contact us to refuse publication

\section{Competing interests}

The authors declare that they have no competing interests.

\section{Publisher's Note}

Springer Nature remains neutral with regard to jurisdictional claims in published maps and institutional affiliations.

\section{Author details}

${ }^{1}$ Department of Psychosomatic Medicine, Faculty of Medicine, Kindai University, 377-2, Ohno-higashi, Osaskasayama City, Osaka 589-8511, Japan. ${ }^{2}$ General Internal Medicine, Sakai City Medical Center, 1-1-1 Ebaraji-cho, Nishi-ku Sakai, Osaka 593-8304, Japan. ${ }^{3}$ Department of Psychosomatic Medicine, Sakai Hospital, Kindai University, 2-7-1, Harayamadai, Minami-ku Sakai, Osaka 590-0132, Japan.

Received: 28 November 2017 Accepted: 19 February 2018

Published online: 13 March 2018

References

1. The Japanese society of psychosomatic medicine, editor. The handbook for the certification of the Japanese society of psychosomatic medicine. Tokyo: the Japanese society of psychosomatic medicine; 1996.

2. Graber ML, Carlson B. Diagnostic error: the hidden epidemic. Physician Exec. 2011;37(6):12-4. 16, 18-9

3. Graber M. Diagnostic errors in medicine: a case of neglect. Jt Comm J Qual Patient Saf. 2005:31(2):106-13.

4. Graber ML, Franklin N, Gordon R. Diagnostic error in internal medicine. Arch Intern Med. 2005;165(13):1493-9.

5. Norman GR, Eva KW. Diagnostic error and clinical reasoning. Med Educ. 2010;44(1):94-100. https://doi.org/10.1111/j.1365-2923.2009.03507.x.

6. McLaughlin K, Eva KW, Norman GR. Reexamining our bias against heuristics. Adv Health Sci Educ Theory Pract. 2014;19(3):457-64. https://doi.org/10. 1007/s10459-014-9518-4. Epub 2014 Jun 3

7. Norman G, Young M, Brooks L. Non-analytical models of clinical reasoning: the role of experience. Med Educ. 2007:41(12):1140-5. Epub 2007 Nov 13

8. Phua DH, Tan NC. Cognitive aspect of diagnostic errors. Ann Acad Med Singap. 2013;42(1):33-41.

9. Norman G. Dual processing and diagnostic errors. Adv Health Sci Educ Theory Pract. 2009;14(Suppl 1):37-49. https://doi.org/10.1007/s10459-009-9179-x. Epub 2009 Aug 11

10. Graber ML, Kissam S, Payne VL, Meyer AN, Sorensen A, Lenfestey N, et al. Cognitive interventions to reduce diagnostic error: a narrative review. BMJ 
Qual Saf. 2012;21(7):535-57. https://doi.org/10.1136/bmjqs-2011-000149. Epub 2012 Apr 27

11. Croskerry P. The importance of cognitive errors in diagnosis and strategies to minimize them. Acad Med. 2003;78(8):775-80.

12. van den Berge $K$, Mamede $S$. Cognitive diagnostic error in internal medicine. Eur J Intern Med. 2013;24(6):525-9. https://doi.org/10.1016/j.jjim.2013.03.006. Epub 2013 Apr 6

13. Ely JW, Graber ML, Croskerry P. Checklists to reduce diagnostic errors. Acad Med. 2011;86(3):307-13. https://doi.org/10.1097/ACM.0b013e31820824cd.

14. Schiff GD, Kim S, Abrams R, Cosby K, Lambert B, Elstein AS, et al. In: Henriksen K, Battles JB, Marks ES, Lewin DI, editors. Diagnosing Diagnosis Errors: Lessons from a Multi-institutional Collaborative Project. Advances in Patient Safety: From Research to Implementation (Volume 2: Concepts and Methodology). Rockville: Agency for Healthcare Research and Quality; US; 2005. p. 255-78

15. Trowbridge RL. Twelve tips for teaching avoidance of diagnostic errors. Med Teach. 2008;30(5):496-500. https://doi.org/10.1080/01421590801965137.

16. Katakami H, Ishikawa E, Hidaka H, Ushiroda Y, Yamaguchi H, Yonekawa T, et al. Clinical feature, incidence, and prevalence of isolated ACTH deficiency (IAD). ACTH related Peptides. 2007;18:29-32. [Japanese]

17. Morigaki Y, Iga J, Kameoka N, Sumitani S, Ohmori T. Psychiatric symptoms in a patient with isolated adrenocorticotropin deficiency: case report and literature review. Gen Hosp Psychiatry. 2014;36(4):449.e3-5. https://doi.org/ 10.1016/j.genhosppsych.2014.02.012. Epub 2014 Mar 5

18. Margolis RL. Nonpsychiatrist house staff frequently misdiagnose psychiatric disorders in general hospital inpatients. Psychosomatics. 1994;35(5):485-91.

19. Juergens SM, Rome JD, llstrup DM. Effect of inpatient psychiatry training on internal medicine residents. Results of a survey Gen Hosp Psychiatry. 1990;12(2):124-8.

20. Andersen SM, Harthorn BH. Changing the psychiatric knowledge of primary care physicians. The effects of a brief intervention on clinical diagnosis and treatment. Gen Hosp Psychiatry. 1990;12(3):177-90.

21. Berner ES, Graber ML. Overconfidence as a cause of diagnostic error in medicine. Am J Med. 2008;121(5 Suppl):S2-23. https://doi.org/10.1016/j. amjmed.2008.01.001

22. Saravay SM, Koran LM. Organic disease mistakenly diagnosed as psychiatric. Psychosomatics. 1977;18(2):6-11.

23. Lucchiari C, Pravettoni G. The role of patient involvement in the diagnostic process in internal medicine: a cognitive approach. Eur J Intern Med. 2013;24(5):411-5. https://doi.org/10.1016/j.ejim.2013.01.022. Epub 2013 Feb 17

24. Schuepbach WM, Adler RH, Sabbioni ME. Accuracy of the clinical diagnosis of 'psychogenic disorders' in the presence of physical symptoms suggesting a general medical condition: a 5-year follow-up in 162 patients. Psychother Psychosom. 2002;71(1):11-7.

\section{Submit your next manuscript to BioMed Central and we will help you at every step:}

- We accept pre-submission inquiries

- Our selector tool helps you to find the most relevant journal

- We provide round the clock customer support

- Convenient online submission

- Thorough peer review

- Inclusion in PubMed and all major indexing services

- Maximum visibility for your research

Submit your manuscript at www.biomedcentral.com/submit

) Biomed Central 